\title{
OPTIMISATION OF TEMPERATURE FIELDS OF MICROSYSTEMS WITH SELF-ORGANISING NEURAL NETS
}

\author{
P. BRATEK and A. KOS* \\ Institute of Electronics, U niversity of M ining and Metallurgy, Mickiewicza 30, \\ 30-059 Kraków, Poland
}

(Received 10 September 2002; In final form 17 November 2002)

\begin{abstract}
Thermal modelling and optimisation of parameter distributed systems is a rather time-consuming process. In this paper the problem of optimisation of temperature fields of VLSI circuits and systems is attacked by a selforganising neural net. The net directly solves the task generated by a heuristic algorithm. No physical model of thermal phenomena is used. The proposed method is simple. Some examples and statistical results are presented. The proposed method is addressed mostly to large, high-speed system designs.
\end{abstract}

Keywords: Thermal problems; Temperature fields optimisation; Neural nets; Microsystems; Topography optimisation

\section{INTRODUCTION}

Due to the rather small thermal conductivity of substrates of VLSI systems, heat dissipated in semiconductor structures may be a reason for the strong electrical feedback between components [1]. The aim of this work is to find such a placement of heat sources (power units, e.g. groups of transistors, integrated parts of processors, power structures in SmartPower systems, etc.) on a chip which leads to flat temperature distribution on the chip. Such a placement guarantees the minimisation of thermo-mechanical stresses, and minimisation of extreme temperatures on the substrate [2]. As a consequence the reliability of the circuit increases [3]. This problem was attacked in some papers [4-6]. A new approach based on a self-organising neural net idea [7], and a heuristic method [8] is proposed in this work. The heuristic approach is based on a simple idea: each heat source of a microcircuit is placed in the centre of gravity of an area proportional to the power dissipated in the heat source. The areas are separable and their sum covers the whole area of a substrate of the circuit. The algorithm is as follows: the substrate is divided into a number $n$ of equal subareas (cells). If $P$ is the total dissipated power, an amount $\mathrm{P} / \mathrm{n}$ is attributed to each subarea. It is necessary that $\mathrm{n}$ be sufficiently large that all the powers $P_{1}, P_{2}, \ldots, P_{n}$ of the heat sources are integer multiples of $P / n$. If $P_{1}=P / n$, for example, a single subarea is chosen and heat source 1

\footnotetext{
* Corresponding author. Tel.: +48-126173435; Fax: +48-126332398; E-mail: kos@ uci.agh.edu.pl
} 


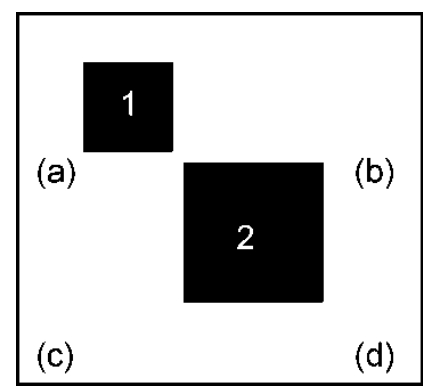

FIGURE 1 Example with two heat sources on the substrate. Power dissipated in 2 is 3 times bigger than in 1 . Source 2 is placed in the centre of gravity of three adjacent cells. $(\mathrm{a})=$ area of source $1,(\mathrm{~b})+(\mathrm{c})+(\mathrm{d})=$ area of source 2 .

will be placed in the centre of the substrate. If $P_{2}=3 P / n$, three neighbouring subareas are grouped together and heat source 2 is placed in the gravity centre of this group. There are, of course, several possibilities to make clusters of subareas. It can be illustrated in Fig. 1 for a simple example and Fig. 2 for a larger number of heat sources.

From the physical point of view it is clear that the areas should resemble hot spots i.e. circles [4]. It means their perimeters should have minimum length. If one perimeter decreases, the others increase. Here one faces the well-known NP-problem. As the number of heat sources increases, the number of possible solutions increases significantly.

To solve that, an approach based on a self-organised neural net [7] is proposed. For simplification of a description it is assumed that every heat source is marked with a separate colour (feature in general) and has the number of his own cells, so called subsources. The number is proportional to the value of power dissipated in the source. The subsources are also identified by their own common colour. The neural net works as follows: subsources are gathered together according to their common features. The subsources having the same colour create only one area attributed to their heat source. One distinguishable subsource in a
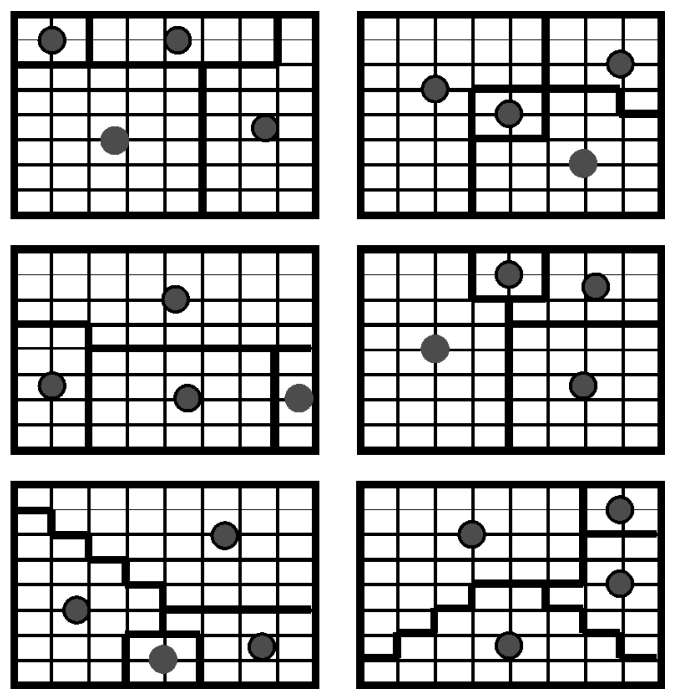

FIGURE 2 Substrate division chosen out of dozens of cases. Placement of four heat sources dissipating $0.4,1,2$ and 3 Watts 
set of subsources of the same colour gathers other cells around itself. In conclusion, the areas created by the same colour subsources should be proportional to the power dissipated in their heat source, and resemble a circle. To get the shape of areas similar to hot spots the sum of distances from subsources having the same colour to their distinguished subsource are minimised. The neural algorithm is described in Section 2.

The motivation of using neural nets is that the abilities of the human brain are still much higher than the abilities of the fastest and most powerful computer ever created. This encourages people to search for new ways of data holding and processing based on the current knowledge of the behaviour of biological brains. $M$ aybe that is why the interest in artificial neural networks has grown so rapidly over the last decade. The most important advantage is that the networks are able to hold and process information in an associative manner. It means that items of information interweave with one another and are processed in a parallel way. The proposed approach does not require any physical models of thermal phenomena taking place in powered integrated circuits and systems.

\section{NEURAL ALGORITHM}

The algorithm consists of two main stages. The first results in a rough placement of heat sources on a substrate. The outcome of the second stage is coherent areas with minimal perimeters attributed to each heat source. The areas are proportional to the powers dissipated in each heat source. Finally the sources are placed in the centres of gravity of their own areas. Initial Data: Iateral expansions of the substrate, number of heat subsources, powers attributed to each subsource.

\subsection{Stage I (Pre-processing)}

At this stage the number of input nodes of the neural net (Fig. 3) equals approximately $25 \%$ of the number of output nodes.

Step 1 Create connectivity matrices. The connectivity matrices $C_{i}(i=1, \ldots, N$ - number of heat sources) are created in the following manner:

(1) For every two cells $i, j$, having a common side $c_{k}(i, j)=1$, otherwise $c_{k}(i, j)=$ $0, \mathrm{k}=1, \ldots, \mathrm{N}$.

(2) No self-connections exist, i.e. $c_{k}(i, i)=0$.

(3) Dimension of $C_{i}$ equals the number of subsources attributed to kth heat source.
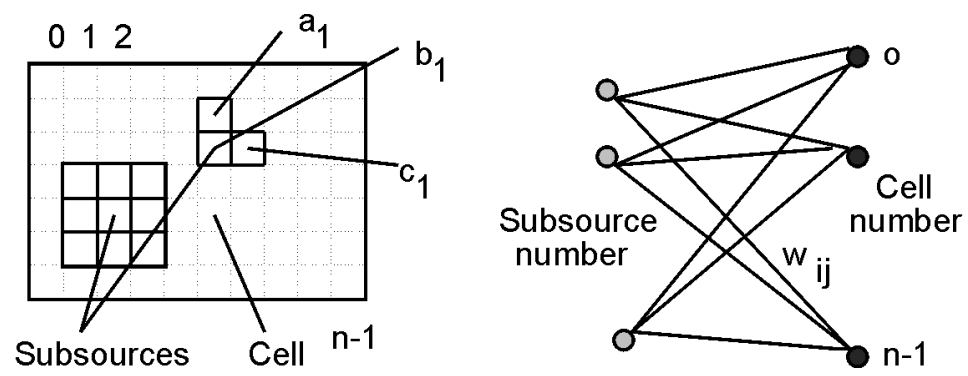

FIGURE 3 Substrate and self-organising neural net. 
Let us take into consideration an example. The heat source, represented by three subsources $a_{1}, b_{1}, c_{1}$ (Fig. 3), has the following connectivity matrix:

$$
C_{1}=\left[\begin{array}{lll}
0 & 1 & 0 \\
1 & 0 & 1 \\
0 & 1 & 0
\end{array}\right]
$$

The connectivity matrix does not include information about connections of subsources via intermediate subsources. To find such strengths of connections, modified connectivity matrices (strength matrices) $C_{i}^{\prime}$ are introduced. For the example mentioned above the strength matrix has the following form:

$$
C_{i}^{\prime}=\left[\begin{array}{ccc}
0 & 1 & 0.5 \\
1 & 0 & 1 \\
0.5 & 1 & 0
\end{array}\right]
$$

Step 2 Create input data. The columns of matrices $C_{i}$ are used as input sequences for the neural net, input_sequence $=$ input_sequence_for_k, where input_sequence_for_k = $<k,|1| 2,, \ldots,|m>;| 1,|2, \ldots|$,$m - numbers of subsources connected to k$ in the following manner:

$$
c_{i}^{\prime}(k, \mid 1) \geq c_{i}^{\prime}(k, \mid 2) \geq \ldots \geq c_{i}^{\prime}(k, I m) .
$$

Step 3 Initialise weights of the neural net. For every input node k, and every output node I a small random value of the weight $w(k, I)$ is applied.

Step 4 Do until convergence.

Step 4.1 New input.

Step 4.2 Find the optimum placement I* for kth input. The following cost function is minimised:

$$
\text { cost_for_k }=\sum_{i=0}^{i=N_{j}^{\prime}-1} c_{j}^{\prime}(k, i) \cdot d_{j}(k, i)
$$

where $d_{j}(k, i)=$ distance between cells, $N_{j}^{\prime}=$ number of subsources for $j$ th source $(\mathrm{j}=1, \ldots, \mathrm{N})$

Condition (3) can be rewritten in such a way that the response function (response of input $\mathrm{i}$ at output node I ) is maximised:

$$
\operatorname{response}(k, l)=\sum_{i=0}^{i=N_{j}^{\prime}-1} c_{j}^{\prime}(k, i) \cdot w_{j}(i, l)
$$

Step 4.3 Adjust weight. For every output node $I \in N E_{t}, I \neq I^{*}$ :

$$
w(k, l)[t+1]=w(k, l)[t]+\gamma_{1}\left(\frac{1}{d\left(l, l^{*}\right)}-w(k, l)[t]\right) .
$$


For $\mathrm{I}=\mathrm{I}^{*}$ :

$$
w(k, l)[t+1]=w(k, l)[t]+\gamma_{2} \frac{\text { number_of_subsources_placed }}{\text { total_number_of_subsources }}(1-w(k, l)[t])
$$

where $\mathrm{t}=$ iteration, $\gamma_{1}, \gamma_{2}=$ coefficients, $N E_{1}=$ neighbourhood of node $\mathrm{I}, \mathrm{I}^{*}=$ the best responding node.

If the solution is not found, go to Step 4.1.

Stage I results in a rough shape of every subarea attributed to its heat source. The centres of gravity of every subarea create convergence points. In the next stage subsources attributed to proper heat sources converge around their own convergence points.

\subsection{Stage II (M ain)}

This stage leads to the division of the substrate into characteristic regions, proportional to powers dissipated in each heat source. The numbers of input and output nodes of the neural network are the same (Fig.3).

Step 1 Attribute proper area of the substrate to every heat source. It is done according to the following formula:

number_of_subsources[0 $[1, \ldots, N-1]=(\operatorname{power}[0,1, \ldots, N-1] *$ area $) /$ total_power

where area $=$ area of substrate, power $=$ value of power dissipated in ith heat source, total_power $=$ total power dissipated in the substrate.

Subsources attributed to various heat sources are indicated with different colours.

Step 2 Initialise weights. Every weight is set to a small random value.

Step 3 Find the output node which best fits the input value. It will be achieved by computing the minimum of the following expression:

$$
\text { (input(input_node) - w(input_node, output_node) })^{2} \text {. }
$$

Step 4 Adapt weights. In this step the weights are adapted in the neighbourhood of node j* by the formula:

$$
w(i, j)[t+1]=w(i, j)+\eta(t)(x(i)-w(i, j)[t])
$$

for $\mathrm{j} \in \mathrm{NE}_{\mathrm{j}^{*}}(\mathrm{t}), 0 \leq \mathrm{i} \leq$ number_of _subsources -1 where $\mathrm{x}=$ input, $\mathrm{i}=$ number of input node, $\mathrm{j}=$ number of output node, $\eta(\mathrm{t})=$ coefficient decreasing according to the experimental formula:

$$
\eta(\mathrm{t})=\frac{0.55}{\text { iteration }}
$$


$\mathrm{NE}_{j^{*}}=$ neighbourhood of node $\mathrm{j}^{*}$. The greater the number of iteration, the smaller the neighbourhood.

Step 5 Go to Step 3 or stop. In the next section hypothetical tasks are solved with the neural algorithm.

\section{EXAMPLES}

The following parameters of the algorithm were assumed: maximal number of heat sources $=10$, maximal number of subsources $=2500, \gamma_{1}=0.01, \gamma_{2}=0.2$.

\subsection{Example I}

Let us take into consideration a silicon substrate with lateral expansions: $30 \times 40 \mathrm{~mm}$, thickness $0.6 \mathrm{~mm}$, cooled by free air. Two heat sources dissipate power $1 \mathrm{~W}$ and $2 \mathrm{~W}$, respectively. The lateral expansion of each heat source is $5 \mathrm{~mm}$. Figure 4 shows the place-

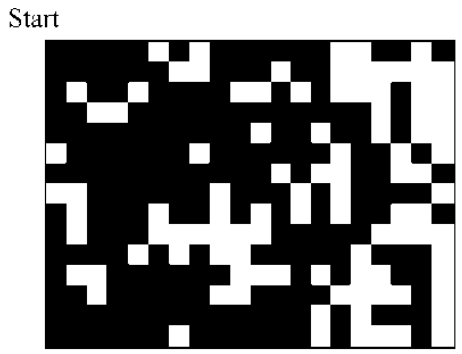

After 30 iterations

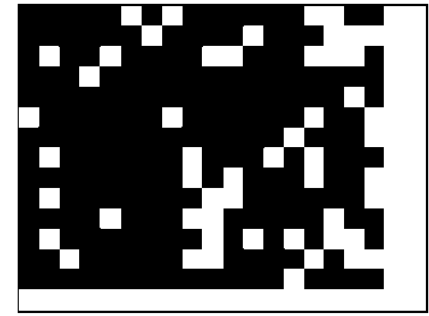

After 45 iterations

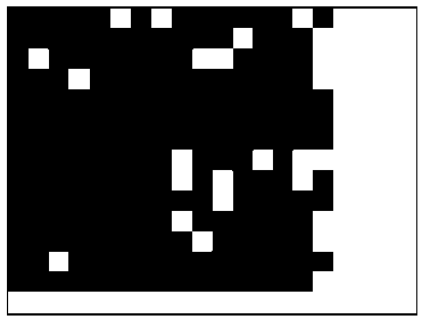

After 54 iterations

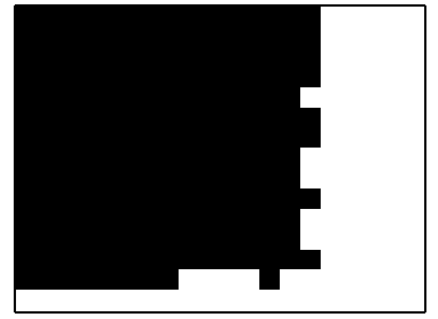

After 61 iterations

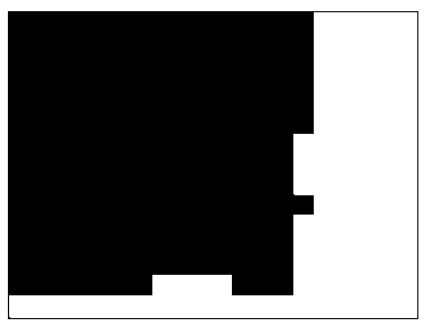

A fler 100 iterations

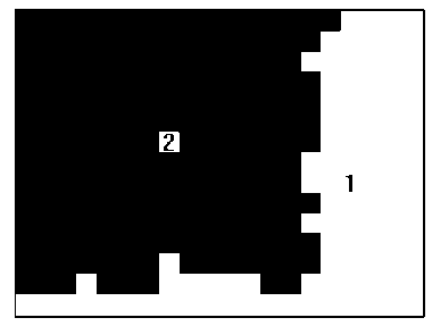

FIGURE 4 Substrate from Example 1. 
ment generated by the neural net. For verification the traditional gradient method was used. The objective function of the optimisation algorithm was the following:

$$
J=\int\left[T(x, y)-T_{m}(x, y)\right]^{2 N} d \xi
$$

where $T_{m}=$ average temperature of the substrate, $\mathrm{N}=$ number of heat sources. The difference between the temperatures of the central points of heat sources obtained by the new technique and the gradient method was less than $5 \%$.

\subsection{Example II}

Three heat sources case ( $1 \mathrm{~W}, 2 \mathrm{~W}, 3 \mathrm{~W}$ ) is presented in Fig. 5. Also for this example the difference between the new technique and the gradient method is less than $5 \%$.

The large number of simulations of heat sources with the different dissipated power was carried out. The results are shown in Fig. 6 and 7.

The yield of the algorithm is defined as a number of successful solutions to the whole number of simulations. The neural computations have been verified by classical tools described in Ref. [2,5].

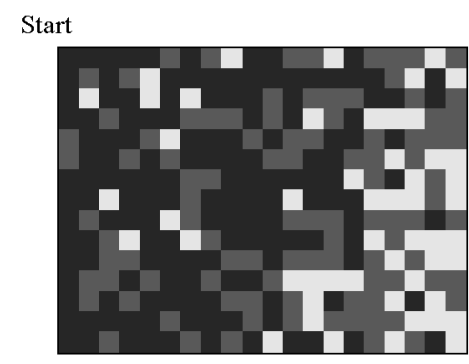

After 45 iterations

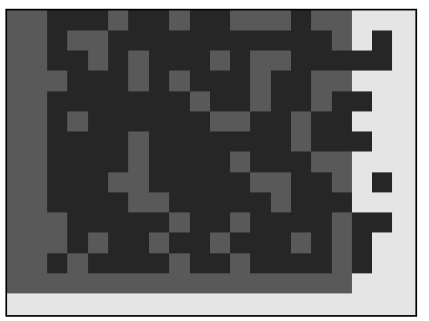

After 61 iterations

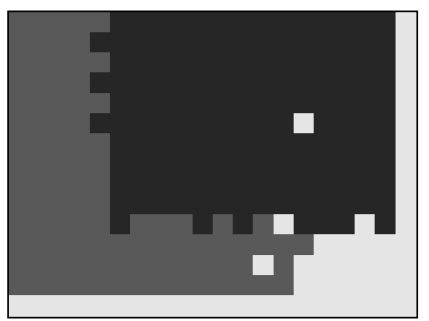

After 25 iterations

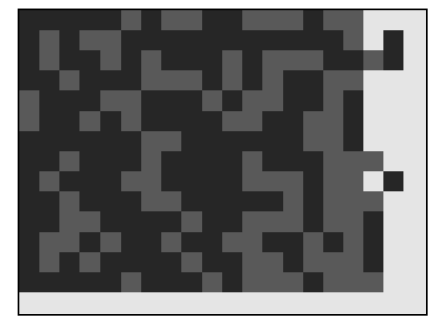

After 55 iterations

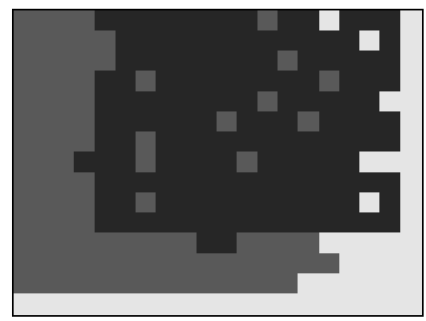

After 100 iterations

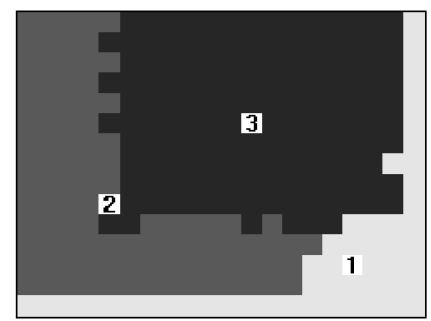

FIGURE 5 Topology from Example 2. 


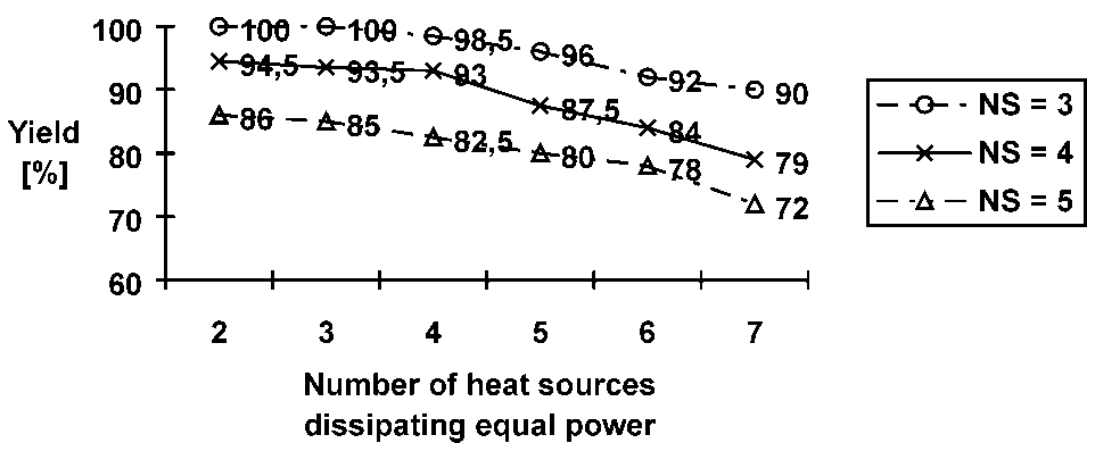

FIGURE 6 Neural algorithm yield versus the number of heat sources. NS = number of subsources creating one heat source.

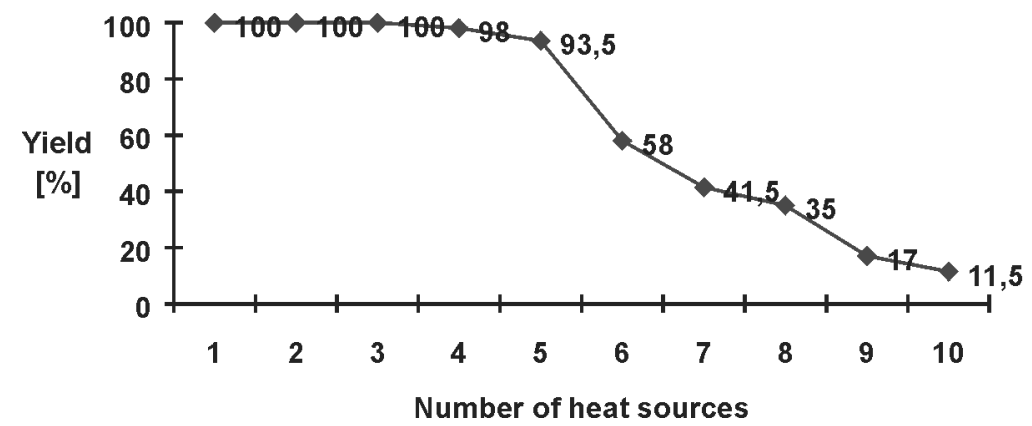

FIGURE 7 The algorithm yield versus the number of heat sources having successively increasing values from 0.1 to 1.0 (e.g. for 3 sources their power values are $0.1,0.2$ and $0.3 \mathrm{~W}$ ).

\section{CONCLUSION}

This paper presents a new idea of an optimisation algorithm dedicated to microcircuits and microsystems topography design in thermal aspect. The algorithm is based on the heuristic method and self-organising neural net. It is much simpler than classical tools, and needs much less time for computation. The algorithm solves up to five heat source problems almost without any mistakes. For much more complicated cases the algorithm can be treated as a generator of quasi-optimum placement, which is a start point for traditional algorithms searching for an optimum solution.

\section{References}

[1] Bhavnagarwala, A. J., A ustin, B. L., Bowman, K. A. and Meindl, J. D. (2000). A minimum total power methodology for projecting limits on CMOS GSI. IEEE Transaction on Very Large Scale Integration (VLSI) Systems, 8(3), 235-251.

[2] Kos, A. and De M ey, G. (1997). Thermal Modelling and Optimisation of Power Microcircuits. Electrochemical Publications, Bristol, England.

[3] M audgal, V. K. (1991). Computer-aided thermal analysis. Hybrid Circuit Technology, 19-21.

[4] Rottiers, L. and De M ey, G. (1988). Hot spot effects in hybrid circuits. IEEE Trans. on Components, H ybrids, and M anufacturing Technology, 11(3), 274-278. 
[5] White, J. (1991). O ptimisation of thin film hybrids using Taguchi methods. H ybrid Circuits Technology, 26-29.

[6] Nakayama, W. (1988). Thermal management of electronic equipment: A review of technology and research topics. In: B ar-Cohen, A and K raus, A. D. (Eds.), Advances in Thermal M odeling of Electronic C omponents and Systems, Vol. 1, Hemisphere Publishing Corp., N ew York.

[7] Kohonen, T. (1991). Self-organizing Maps: Optimization Approaches, Artificial Neural Networks. Elsevier Science Publishers B.V., pp. 981-990.

[8] Kos, A . and De M ey, G. (1994). Thermal placement in hybrid circuits - a heuristic approach. Active and Passive Electronic Components, 17, 67-77. 

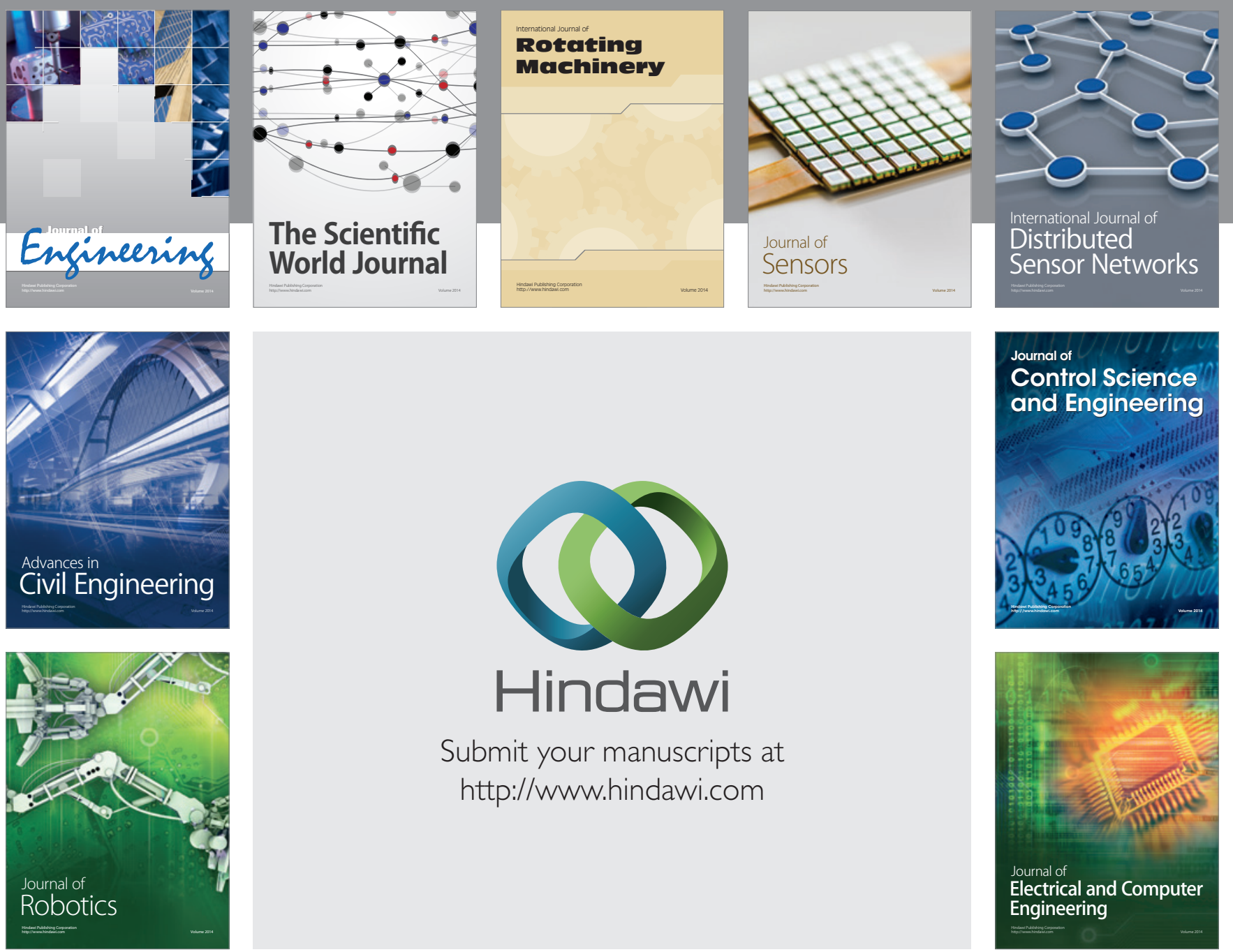

Submit your manuscripts at

http://www.hindawi.com
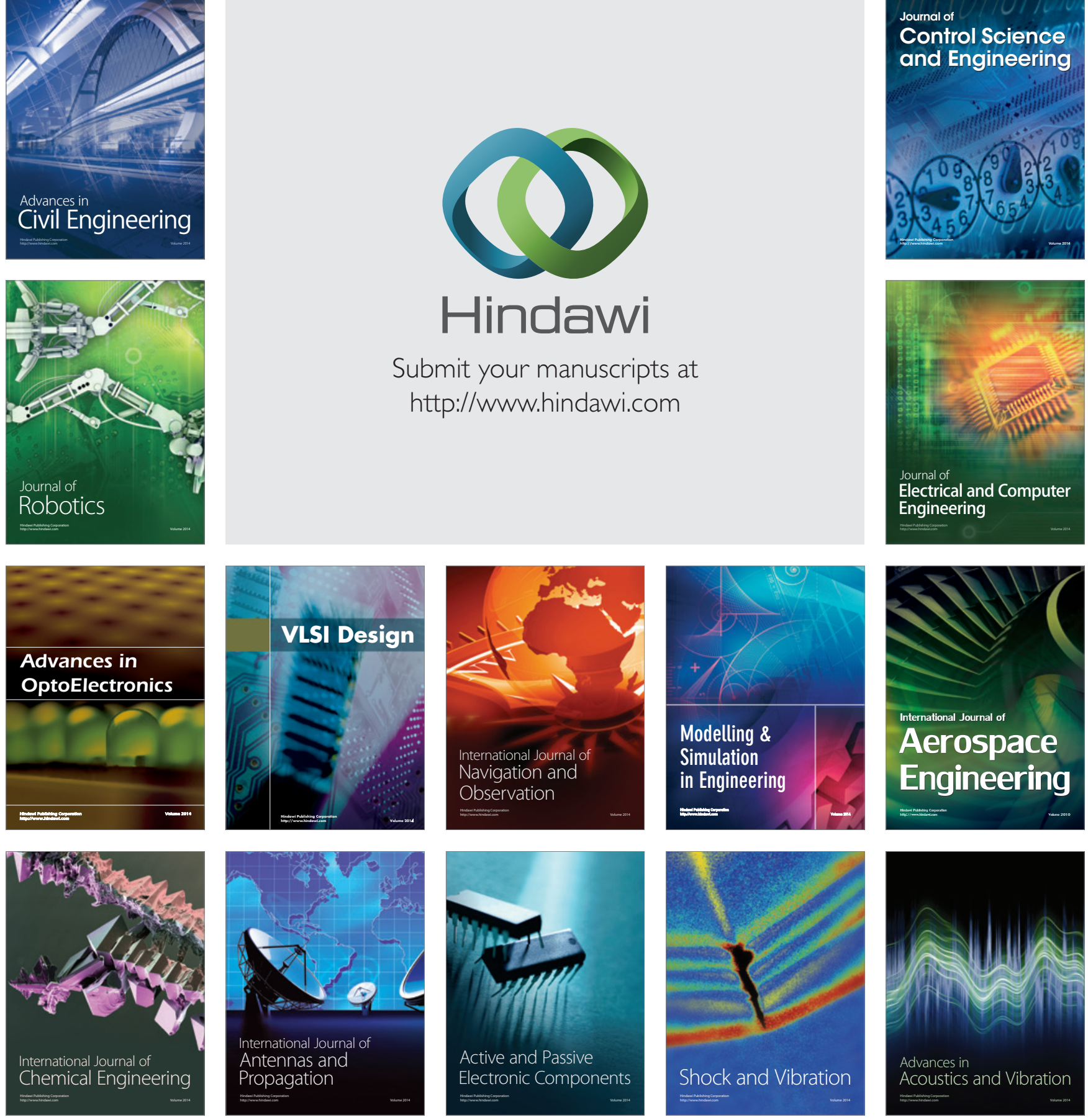